\title{
Two-Photon Microscopy Of Archived Human Nephrectomy Specimens
}

\author{
M.P. Goheen*, E.C. Chambers**, C.L. Phillips*
}

Pathology* and Medicine/Nephrology**, Indiana University School of Medicine and the Indiana Center for Biological Microscopy, 950 W. Walnut R2-202, Indianapolis, IN 46202, USA

Two-photon fluorescence microscopy and volume rendering software permit three-dimensional (3D) exploration of structures embedded within thick biological specimens. Hospital-based human tissue banks are a rich source of biopsy and surgical specimens for morphological analysis, but preservation of 3-D tissue architecture may be compromised by suboptimal fixation and storage conditions, i.e., archival in formaldehyde-based fixatives. Using techniques we have previously established for animal studies [1-3], we sought to determine optimal fixation conditions and fluorescent markers that would permit 3-D analysis of tissue architecture in human nephrectomy specimens archived in readily available hospital-based fixative solutions. Wedges of normal human kidney were retrieved from surgical nephrectomy specimens and fixed for 48 hours in $4 \%$ paraformaldehyde (PFA) prepared fresh and either frozen at $-20^{\circ} \mathrm{C}$ or stored for 6 months at $25^{\circ} \mathrm{C}$, buffered with $1 \mathrm{x}$ phosphate buffered saline (PBS) or $0.15 \mathrm{M}$ sodium cacodylate. Comparison was made to kidney fixed in 10\% neutral buffered formalin (NBF, traditional brightfield microscopy fixative) or 3\% glutaraldehyde/ $0.15 \mathrm{M} \mathrm{NaCacodylate} \mathrm{(traditional} \mathrm{electron} \mathrm{microscopy} \mathrm{fixative).}$ Using Bio-Rad and Zeiss two-photon microscopes at the Indiana Center for Biological Microscopy (www.nephrology.iupui.edu/imaging), optical Z-series were collected with a 20x water-immersion objective from 200 um-thick vibratome tissue sections incubated with fluorescent peanut agglutinin (labels proximal tubules and collecting ducts), Dolichos biflorus lectin (collecting ducts), phalloidin (F-actin) and/or DAPI (nuclei). Volumes were rendered in 3-D with Voxx (freeware, www.nephrology.iupui.edu/imaging/voxx/index.htm) or Amira software. Images were collected and visually analyzed by investigators 'blinded' to fixative solutions (ranked in order of 1/best to 5/worst). While all samples contained cellular elements that could be imaged and visually identified (e.g., glomerular capillary loops, apical brush border of proximal tubules and nuclei), we found no difference in glomerular (figure 1) or tubular (figure 2) architecture in tissue fixed in variously aged PFA, whether buffered with PBS or sodium cacodylate (all scored as 1). Samples fixed in PFA, regardless of buffer, showed overall better architectural preservation as compared to NBF (scored as 4) or glutaraldehyde (scored as 5). In conclusion, we found that human kidney specimens fixed in readily available hospital-based fixation solutions could be retrieved for 3-D image analysis by twophoton microscopy. Overall tissue architecture was sufficiently preserved when viewed with a $20 \mathrm{x}$ objective. This technology, combined with selective fluorescent markers and volume rendering software, provides useful information on overall 3-D tissue architecture with utility for morphological analysis of normal structures and histopathological lesions found in human surgical pathology specimens. Therefore, "wet" specimens stored in hospital-based tissue banks are not limited to analysis with conventional paraffin-embedding and two-dimensional histological stains.

\section{References}

[1] C.L. Phillips et al., Amer J Pathol 158: 49-55 (2001)

[2] J.L. Clendenon et al., Am J Physiol Cell Physiol 282: C213 (2002)

[3] C.L. Phillips et al., J Am Soc Nephrol. 15(7):1744-55 (2004) 
Human kidney by 2-photon microscopy, 20x water objective, pixel size $0.96 \times 0.96 \times 1.00$ channel 1 phalloidin-rhodamine (brown) channel 2 blank

channel 3 DAPI (blue)
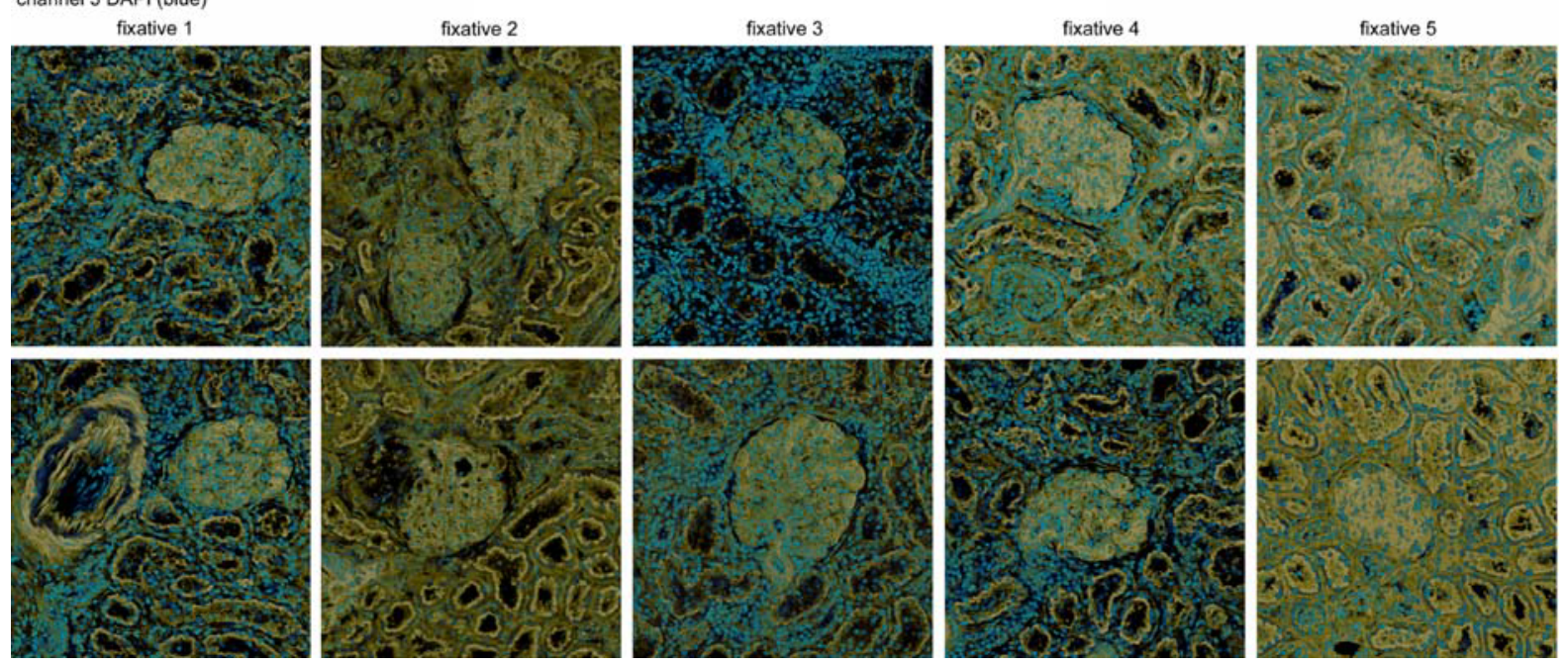

Figure 1: Two-photon microscopy and 3-D rendering of human kidney. Tissue wedges were retrieved from surgical nephrectomy specimens, procured away from tumor, and fixed for 48 hrs in: Fixative 1: 4\% paraformaldehyde/ $0.15 \mathrm{M} \mathrm{NaCacodylate} \mathrm{stored} \mathrm{for} 6$ months at $25^{\circ} \mathrm{C}$, Fixative 2: $3 \%$ glutaraldehyde/ $0.15 \mathrm{M} \mathrm{NaCacodylate}$ prepared fresh, stored at $-20^{\circ} \mathrm{C}$ then thawed, Fixative 3: 4\% paraformaldehyde/1xPBS prepared fresh and stored at $-20^{\circ} \mathrm{C}$ then thawed, Fixative 4: $10 \%$ neutral buffered formalin (hospital grade from commercial source) stored at $25^{\circ} \mathrm{C}$, Fixative 5: 4\% paraformaldehyde $/ 0.15 \mathrm{M} \mathrm{NaCacodylate} \mathrm{prepared} \mathrm{fresh,} \mathrm{stored} \mathrm{at}-20^{\circ} \mathrm{C}$ then thawed Vibratome sections were labeled with DAPI (nuclei, pseudocolored blue) and phalloidin (F-actin, brown).

Human kidney by 2-photon microscopy, 20x water objective, pixel size $0.96 \times 0.96 \times 1.00$

channel 1 rhodamine peanut agglutinin (red)

channel 2 fluorescein Dolichos biflorus (green)

channel 3 DAPI (blue)

fixative 1

fixative 2

fixative 3
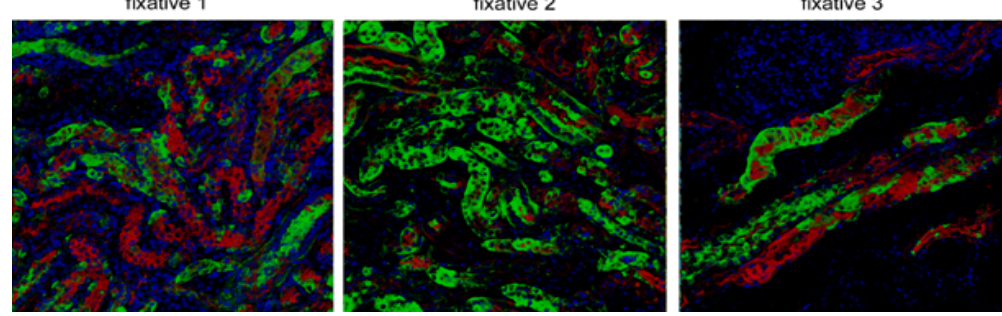

fixative 4

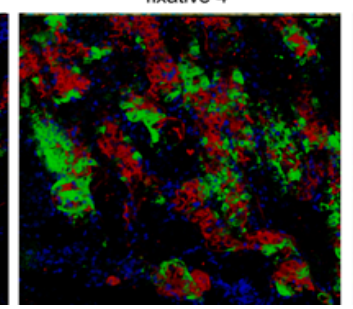

fixative 5

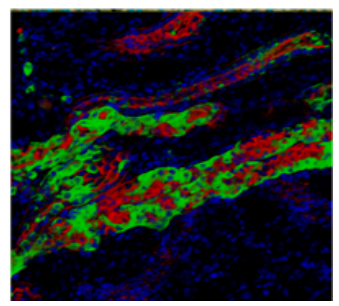

Figure 2: Two-photon microscopy and 3-D rendering of human kidney. Normal human kidney was procured, fixed and processed as in figure 1. Vibratome sections were labeled with DAPI (nuclei, blue), peanut agglutinin lectin (red) and Dolichos biflorus lectin (green).

Prior approval for research on human tissues was obtained from the Institutional Review Board at Indiana University School of Medicine. 